Leitthema

Unfallchirurg $2020 \cdot 123: 830-835$

https://doi.org/10.1007/s00113-020-00895-3

Online publiziert: 16 . Oktober 2020

๑ Springer Medizin Verlag GmbH, ein Teil von

Springer Nature 2020

\section{Redaktion}

D. A. Back, Berlin

D. Pförringer, München

Dominik Pförringer ${ }^{1}$ Jörg Ansorg ${ }^{2} \cdot$ Georg Osterhoff ${ }^{3}$. Florian Dittrich ${ }^{4}$. Julian Scherer ${ }^{5}$. Uwe de Jager ${ }^{6}$. AG Digitalisierung DGOU, BVOU · David A. Back

${ }^{1}$ Klinik und Poliklinik für Unfallchirurgie, Klinikum rechts der Isar, TU München, München, Deutschland

${ }^{2}$ Berufsverband für Orthopädie und Unfallchirurgie e. V. (BVOU e. V.), Berlin, Deutschland

${ }^{3}$ Klinik und Poliklinik für Orthopädie, Unfallchirurgie und Plastische Chirurgie, Universitätsklinikum Leipzig AöR, Leipzig, Deutschland

${ }^{4}$ Klinik und Poliklinik für Orthopädie, Universität des Saarlandes, Homburg, Deutschland

${ }^{5}$ Klinik für Traumatologie, UniversitätsSpital Zürich, Zürich, Schweiz

${ }^{6}$ Orthopädische Praxis Dr. Uwe de Jager, Freudenstadt, Deutschland

${ }^{7}$ Berlin, Deutschland

${ }^{8}$ Klinik für Unfallchirurgie und Orthopädie, Bundeswehrkrankenhaus Berlin, Berlin, Deutschland

\title{
Digitalisierung in Orthopädie und Unfallchirurgie: Stand 2020 in Klinik und Praxis
}

\section{Telematikinfrastruktur und elektronische Gesundheitskarte}

\section{Entwicklung und Einführung}

Modernisierung der gesetzlichen Krankenversicherung" aus dem Jahr 2003 ist auch die ärztliche Selbstverwaltung aus Kassenärztlicher Bundesvereinigung $(\mathrm{KBV})$ und Bundesärztekammer in dieses umfangreiche Projekt durch ihre Beteiligung an der Gesellschaft für Telematikanwendungen der Gesundheitskarte (GEMATIK) eingebunden. Neben der Optimierung von Behandlungsprozessen und Patientenversorgung ist mit der Digitalisierung des Gesundheitssystems v.a. die Hoffnung verbunden, die Versorgungssektoren miteinander zu vernetzen und die in den letzten Jahrzehnten betonierte Sektorengrenze für Informationen und Optimierungsprozesse durchlässiger zu gestalten. Nach 20 Jahren Projektarbeit und Milliardeninvestitionen sollen in diesem Beitrag wesentliche aktuelle Projekte und deren Status reflektiert sowie konkrete Anwendungsszenarien und erste Umsetzungsbeispiele für Orthopädie und Unfallchirurgie (U und O) aufgezeigt werden.
Rückgrat für die digitale Kommunikation zwischen Ärzten, Praxen und Kliniken sowie Apotheken und weiteren Gesundheitsberufen ist eine sichere digitale Vernetzung. Damit sollen medizinische Informationen für eine effizientere Patientenbehandlung schneller und vollständiger als im analogen Zeitalter verfügbar und zugänglich gemacht werden. Die Telematikinfrastruktur (TI) wurde gemeinsam mit der ersten Version der elektronischen Gesundheitskarte (eGK) seit 2005 von der GEMATIK entwickelt und wird seit 2018 eingeführt. Für den Anschluss an die TI sind ein Konnektor sowie ein Kartenterminal für jede Praxis erforderlich. Vertragsärzte und Praxen, die sich nicht an die TI anschließen, werden per Gesetz mit Sanktionen belegt. Neben Lieferengpässen war die Einführungsphase von Verunsicherung und Sicherheitsbedenken aufseiten der Ärzte geprägt [1]. Beim Anschluss an die TI [2] kam auf, dass die IT-Infrastruktur vieler Praxen erhebliche Sicherheitsmängel aufweist [3]. Hinzu kamen Mängel beim Ausgabeverfahren der Authentifizierungskarten $[4,5]$.
Die KBV wurde vom Gesetzgeber beauftragt, bis zum 30.06.2020 eine Richtlinie zur IT-Sicherheit in Arztpraxen zu entwickeln, die die Praxen bei korrekter Umsetzung rechtlich absichern soll. Das Versichertendatenmanagement (VSDM) ist bislang die einzige verfügbare Anwendung der TI [6]. Es dient der Stammdatenaktualisierung und bietet Praxen wenig Mehrwert im Alltag.

\section{Sinnvolle Anwendungen}

Alle für Praxisablauf und Effizienzsteigerung der ärztlichen Kommunikation sinnvollen Anwendungen seitens der TI und eGK sind aktuell noch nicht verfügbar. Die GEMATIK und die KBV arbeiten an konkreten Spezifikationen für die einzelnen Anwendungen sowie an deren Einführung. Alle zukünftigen Anwendungen werden jedoch erst nach einem Software-Update des Konnektors und der Ausgabe neuer elektronischer Heilberufsausweise (eHBA) verfügbar sein. Insofern darf mit weiteren Verzögerungen gerechnet werden.

Beispiele für sinnvolle digitale Anwendungen sind:

- qualifizierte elektronische Signatur (QES),

- Notfalldatenmanagement (NFDM) auf der eGK, 


\section{Infobox 1 Wie erkenne ich eine seriöse App? \\ - Adäquate, seriöse Store-Beschreibung (deutsch/englisch? Zweckbeschreibung verständlich? Funktionen plausibel?) \\ - Release-Datum (Upgrade) \\ - Informationen zu App und Hersteller \\ - Homepage/Impressum \\ - Datenschutzerklärung (verständlich? App ohne Dateneingabe nutzbar?) \\ - Quellenangaben, wissenschaftliche Belege \\ - Verweis auf Entwickler und deren Hin- tergrund/Qualifikationen, User-Zahlen, Bewertungen, Berichte (Achtung: „Fake- Bewertungen" möglich)}

- elektronischer Medikationsplan (eMP),

- elektronischer Arztbrief (eArztbrief),

- elektronische Arbeitsunfähigkeitsbescheinigung (eAU),

- elektronisches Rezept (eRezept),

- (zentrale) elektronische Patientenakte (ePA).

Die gesetzlichen Krankenversicherungen wurden per Gesetz verpflichtet, ihren Versicherten ab 01.01.2021 eine (zentrale) ePA anzubieten. Um die ePA vonseiten der Ärzte mit Befunden ,füllen" zu können, ist bis Ende 2020 eine Vielzahl von Voraussetzungen (u. a. Konnektor-Update, 2nd Gen eHBA, QES) zu schaffen.

\section{Digitalisierungstrends außer- halb der Telematikinfrastruktur}

Unabhängig von den Digitalisierungsprojekten seitens des Staates und der Selbstverwaltung führen viele Praxen und Kliniken aufgrund der hohen Patientennachfrage bereits eigenständig digitale Angebote ein. Hier ist zum einen die Möglichkeit einer Onlineterminvergabe zu nennen, die zu einer Entlastung der Angestellten im Anmeldungsbereich führen kann. Viele Angebote bieten Komfortfunktionen wie Short-Message-Service(SMS)-Benachrichtigung und Integration der privatärztlichen Verrechnungsstelle (PVS).

Rund um die digitale Terminvergabe können Praxisressourcen wie Räume, Geräte und Personal geplant sowie über die Vernetzung von Praxen und Kliniken Befunde ausgetauscht werden. Mit einem digitalen Praxismanagement können Prozesse in der Praxis optimiert und Ressourcen effizienter genutzt werden [7]. Für Kliniken gilt dies potenziert, da diese an Komplexität in organisatorischen und ökonomischen Aspekten laborieren und oftmals unter veralteten Krankenhausinformationssystemen (KIS) leiden.

Einige weitere Digitalisierungsaspekte im Kontext der Themen Apps, Onlinevideosprechstunde (OVS) und ePA sowie Gedanken zum Datenschutz werden im Folgenden ausführlicher vorgestellt.

\section{Apps und "mobile health"}

Das Smartphone ist unbestreitbar eine der am kontroversesten diskutierten technologischen Errungenschaften des 21. Jh., die mit einer rasanten Geschwindigkeit Einfluss auf große Bereiche unserer Gesellschaft genommen hat. Die Liste der Vor- und auch Nachteile, die mit einer alltäglichen Smartphone-Nutzung einhergehen, ist lang und weitestgehend paritätisch besetzt. Die Vorzüge eines angemessenen Einsatzes von Apps im Bereich der Orthopädie und Unfallchirurgie $(\mathrm{OU})$ sind jedoch unbestreitbar und könnten ein enormes Potenzial für die Bewältigung zukünftiger Herausforderungen im Gesundheitswesen darstellen [16].

Auch politisch wurden mit dem kürzlich ratifizierten Digitale-VersorgungGesetz (DVG) die Weichen hin zu einer flächendeckenden Implementierung von Apps gestellt, u.a. durch die Verschreibung von Gesundheits-Apps auf Rezept. Das DVG schafft im internationalen Vergleich erstmalig einen Leistungsanspruch auf digitale Gesundheitsanwendung, beschränkt auf Medizinprodukte niedriger Risikoklassen.

Die Aufnahme in das Verzeichnis für digitale Gesundheitsanwendungen erfolgt nach Antragstellung durch den Hersteller und Prüfung, ob die Gesundheits-App grundlegenden Anforderungen an Medizinprodukte und Datensicherheit gerecht wird sowie positive Versorgungseffekte aufweist [13]. Eine kritische Auseinandersetzung der Ärz- teschaft mit dem neuen Medium ist obligat.

Apps werden in Deutschland aktuell von $63 \%$ der Orthopäden und Unfallchirurgen regelmäßig im klinischen Alltag eingesetzt. Aufgrund unscharf definierter juristischer, ethischer und medizinischer Regularien bewegen sich viele Apps jedoch in einer Grauzone [15]. Vergangene Datenskandale und eine intransparente Datenverarbeitung haben zu einem Grundmisstrauen gegenüber Apps, die Gesundheitsdaten erheben, geführt [19].

Eine der größten Herausforderungen für deutsche Ärzte in $\mathrm{O}$ und $\mathrm{U}$ stellt bereits die Suche nach der passenden App dar. Die Unübersichtlichkeit und Dynamik der App Stores lässt die Suche nach der passenden App wie die Suche nach der "Stecknadel im Heuhaufen“ erscheinen [14]. Lösungsansätze können die stetig ansteigende Zahl von App-Store-basierten Reviews kommerziell erhältlicher Apps darstellen, die sich kritisch mit den derzeit verfügbaren Apps auseinandersetzen sowie inhaltliche und methodische Ansätze für die persönliche App-Suche liefern [17]. Diese Methoden könnten in Zukunft um automatische algorithmische Analysen oder künstliche Intelligenz erweitert werden.

\section{1) Ein Rezensionsverfahren in Form eines DGOU-App-Siegels ist aktuell in einem Pilottest im Einsatz}

Neben der Suche nach den passenden Apps ist die objektive Evaluation ihrer Sicherheit und Qualität problematisch (- Infobox 1). Verschiedene Testverfahren wurden entwickelt und werden ständig weiterentwickelt. Etablieren konnten sich die auf die Vertrauenswürdigkeit einer App fokussierende „App-Synopsis" des Peter L. Reichertz Instituts für Medizinische Informatik an der Medizinischen Hochschule Hannover [11] und die „Mobile App Rating Scale“ [18], die eine objektive Bewertung des Inhalts und der technischen Spezifikationen einer App ermöglicht. Eine qualitative Verbesserung der Evaluation könnte er- 
reicht werden, wenn zukünftige Ratings adaptiver an die Zweckbestimmung der App anpassbar würden.

Mehrere Fachgesellschaften sowie private Institutionen rezensieren Apps nach einem standardisierten, transparenten und öffentlich zugänglichen Fragenkatalog und veröffentlichen diese Rezensionen in App-Bibliotheken (z.B. https:// www.nhs.uk/apps-library/). Ein entsprechendes Verfahren eines App-Siegels der Deutschen Gesellschaft für Orthopädie und Unfallchirurgie (DGOU) ist auf Grundlage definierter, evidenzbasierter Gütekriterien aktuell in einem Pilottest im Einsatz [12].

Die durch die „coronavirus disease 2019“ (COVID-19) ausgelöste Pandemie hat die Dynamik des App-Markts zusätzlich erhöht. Die Zahl der öffentlich angebotenen vertrauenswürdigen und qualitativ hochwertigen Apps ist aktuell jedoch noch überschaubar. Vielversprechende Ansätze zur Schaffung belastbarer Evidenzen im Kontext von mHealth z.B. systematische Appstore Screening, App-Rating-Entwicklungen, App-Reviews oder App-Libraries sind in Entwicklung befindlich. Es sind jedoch weitere Anstrengungen von $\mathrm{Me}$ dizin, IT und Legislative in Form von sektorübergreifenden Expertengruppen erforderlich, um zu gewährleisten, dass seriöse innovative digitale Gesundheitsanwendungen den Weg in die tägliche klinische Praxis finden.

\section{Onlinevideosprechstunde}

Die Kommunikation von 2 und mehr Personen über Smartphone, Tablet oder PC gehört heute zum Alltag vieler Menschen. Immer mehr Privatpersonen und auch die Mitglieder der betagteren Generation besitzen heutzutage einen Laptop oder ein Smartphone, mit dem eine OVS möglich wäre [20]. Eine Studie aus den USA hat gezeigt, dass $60 \%$ der Orthopäden Telemedizin als wichtiges Tool bewertet haben, und eine Studie der Bertelsmann-Stiftung fand heraus, dass $45 \%$ der Deutschen eine OVS beim Hausoder Facharzt nutzen würden [21, 22]. Seit 2018 ist die ausschließliche Beratung und/oder Behandlung durch Medien wie die OVS erlaubt [8], sofern diese

Unfallchirurg 2020 $\cdot 123: 830-835$ https://doi.org/10.1007/s00113-020-00895-3

(c) Springer Medizin Verlag GmbH, ein Teil von Springer Nature 2020

D. Pförringer · J. Ansorg · G. Osterhoff · F. Dittrich · J. Scherer · U. de Jager · AG Digitalisierung DGOU, BVOU · D. A. Back

\section{Digitalisierung in Orthopädie und Unfallchirurgie: Stand 2020 in Klinik und Praxis}

\section{Zusammenfassung}

Der vorliegende Beitrag behandelt aus Sicht von Orthopädie und Unfallchirurgie den aktuellen Stand Mitte 2020 im klinischen sowie im Praxisumfeld. Es wird auf Risiken, Schwierigkeiten ebenso wie Potenziale und Chancen im Detail eingegangen. Spezifisch werden die folgenden Themenbereiche erörtert: Telematikinfrastruktur, Apps und "mobile health", Onlinevideosprechstunde, elektronische Patientenakte sowie Datenschutz. Es wird auf die Vor- und Nachteile und den jeweils aktuellen Stand im Spezialfall der Orthopädie und Unfallchirurgie eingegangen.
Zudem werden 7 sinnvolle Beispiele aus dem Bereich der digitalen Anwendung genannt. Eine Mitgliederbefragung des Berufsverbandes der Orthopäden und Unfallchirurgen, BVOU, wird beschrieben und analysiert. Im abschließenden Ausblick werden die aktuellen Hürden und künftig zu klärende Themenbereiche adressiert.

Schlüsselwörter

"Mobile health" · Telekommunikationen . Elektronische Patientenakte · Datenschutz . Befragungen

\section{Digitalization in orthopedics and trauma surgery: current status of clinical and practical aspects 2020}

\section{Abstract}

This article deals with the current state mid2020 in the clinical and practical aspects from the perspective of orthopedics and trauma surgery. The risks, difficulties, potentials and options are discussed in detail. The following topics are specifically debated: infrastructure of telematics, apps and mobile health, online video consultation, electronic medical records and data protection. The advantages and disadvantages and the current state of each topic in the special case of orthopedics and trauma surgery are discussed. Additionally, seven meaningful examples from the field of digital applications are named. A survey of members of the Professional Association of Orthopedic and Trauma Surgeons (BVOU) is described and analyzed. In a concluding perspective the current hurdles and future topics that need clarification are addressed.

\section{Keywords}

Mobile health - Telecommunications . Electronic medical records - Data privacy . Surveys ärztlich vertretbar ist, die ärztliche Sorgfalt gewahrt bleibt und der Patient über die Besonderheiten der OVS aufgeklärt wurde.

Einen Boom erlebt die OVS aktuell durch die Coronapandemie. In Zeiten, in denen physische Arztbesuche eher vermieden oder wegen Quarantäne nicht wahrgenommen werden können, sind Telemedizin und insbesondere die OVS geeignete Mittel zur Aufrechterhaltung einer adäquaten Arzt-PatientBeziehung und für eine kontinuierliche Betreuung des Patienten. Die integrierte Videokomponente unterstützt, im Gegensatz zum reinen Telefonat, den persönlichen Kontakt und kann auch zu diagnostischen Zwecken eingesetzt werden. In der $\mathrm{O}$ und $\mathrm{U}$ können so aus der Ferne z.B. Wunden inspiziert, in Rehabilitation befindliche Patienten visitiert, Bewegungsausmaße gemessen und dringende persönliche Fragen beantwortet werden [24]. Durch Einsatz der Videosprechstunde können Gesundheitskosten sowie (Anfahrts-)Kosten gesenkt und eine effizientere Arzt-PatientInteraktion etabliert werden [23].

Erste Vergütungsgrundsätze für die Videosprechstunde wurden im EBM [25] und in der GOÄ [26] verankert und während der Coronakrise aufgewertet. Ziel sollte die äquivalente Vergütung von Videosprechstunde und Patientenberatung in der Praxis sein. Im Rahmen von Selek- 
tivverträgen ist dies in $\mathrm{O}$ und $\mathrm{U}$ bereits heute möglich [27].

Die OVS ist vom Gesetzesgeber gewollt und wird von Ärzten und Patienten nachgefragt [28]. Vor allem in strukturschwächeren Regionen kann die OVS in der O und Uvon Vorteil sein. Die OVS hat erhebliches Potenzial für die Unterstützung der sektorübergreifenden Versorgung. Erste Initiativen zur (Video-)Vernetzung von Kliniken und Praxen laufen bereits.

\section{\) Die äquivalente Vergütung von OVS und Patientenbe- ratung ist im Rahmen von Selektivverträgen möglich}

Auch im Rahmen von Zweitmeinungsverfahren ist die OVS eine interessante Option, mehrere Experten zur Falldiskussion zusammenzubringen. Grundbedingung für den Erfolg solcher Zweitmeinungsprojekte ist, dass alle relevanten $\mathrm{Be}$ funde den Experten digital zur Verfügung gestellt werden. $\mathrm{Zu}$ beachten ist, dass eine ärztliche Konsultation, rein auf einer OVS basierend, niemals eine körperliche Untersuchung ersetzen kann und somit der Einsatz einer OVS verantwortungsvoll abgewogen werden muss.

\section{Elektronische Patientenakte}

Zentraler zukünftiger Baustein der TI ist die ePA. Sie soll sektorenübergreifend die digitale Dokumentation der Krankheitsgeschichte gewährleisten. Spätestens ab Januar 2021 müssen die gesetzlichen Krankenkassen ihren Versicherten die ePA anbieten [29]. Folgende Informationen der Versicherten sollen in der ePA gespeichert werden:

- Befunde,

- Diagnosen,

- Therapiemaßnahmen,

- Behandlungsberichte,

- Impfungen.

Laut KBV ist somit eine fall- und einrichtungsübergreifende Dokumentation möglich. Die ePA unterstützt außerdem den Notfalldatensatz, den eMP sowie eArztbrief. Die ePA ersetzt nicht die
Kommunikation unter den Ärzten oder zu anderen Einrichtungen des Gesundheitswesens [30].

Das Bundesgesundheitsministerium [31] hat am 01.04.2020 den „Entwurf eines Gesetzes zum Schutz elektronischer Patientendaten in der Telematikinfrastruktur“ (Patientendaten-Schutz-Gesetz, PDSG; [32]) verabschiedet, in dem die folgenden Vorstellungen von einer ePA formuliert sind:

- Die Nutzung der ePA ist freiwillig.

Der Versicherte entscheidet, welche

Daten in der ePA gespeichert oder

wieder gelöscht werden. Er entscheidet auch in jedem Einzelfall, wer auf die ePA zugreifen darf.

- Neben Befunden, Arztberichten oder Röntgenbildern lassen sich ab 2022 der Impfausweis, der Mutterpass, das gelbe U-Heft für Kinder und das Zahnbonusheft in der ePA speichern.

- Versicherte können ab 2022 bei einem Krankenkassenwechsel ihre Daten aus der ePA übertragen lassen.

- Ab 2022 sollen Versicherte darüber hinaus die Möglichkeit bekommen, über ihr Smartphone oder Tablet für jedes in der ePA gespeicherte Dokument einzeln zu bestimmen, wer darauf zugreifen kann.

- Wer seine Daten in der ePA einsehen möchte, kann das auf dem eigenen Smartphone oder Tablet tun. Auch Versicherte, die kein mobiles Endgerät besitzen, bekommen die Möglichkeit, ihre ePA z.B. in einer Filiale ihrer Krankenkasse einzusehen. Die Kassen werden verpflichtet, die technische Infrastruktur dafür ab 2022 zur Verfügung zu stellen.

- Ab 2023 haben Versicherte die Möglichkeit, die in der ePA abgelegten Daten freiwillig pseudonymisiert und verschlüsselt der medizinischen Forschung zur Verfügung zu stellen.

Die gesetzlich Versicherten haben mit dem neuen Gesetzentwurf des PDSG einen klar geregelten Anspruch gegenüber Vertragsärzten, Krankenhäusern sowie anderen Leistungserbringern, dass alle für ihre Versorgung relevanten Daten in die Akte übertragen werden. Die Nutzung der Akte selbst soll aber für den Versicherten freiwillig bleiben.
Zunächst ist geplant, dass die zugriffsberechtigten Leistungserbringer alle Daten des Patienten einsehen können, es sei denn, dass der Versicherte sie löscht. Ab 2022 sollen die Versicherten ein abgestimmtes Berechtigungsmanagement erhalten, in dem sie die in der Akte enthaltenden Daten gezielt für einzelne Ärzte und andere Leistungserbringer freischalten können. Der Versicherte ist somit „Herr“ seiner Daten.

Bei den Ärzten herrscht Skepsis, da u.a. eine Löschung von Akteninhalten durch Patienten Ärzten u. U. lebenswichtige Informationen vorenthalten könnte [33]. Im Rahmen einer nichtrepräsentative Onlineumfrage des Berufsverbands für Orthopädie und Unfallchirurgie (BVOU) zur ePA [34] gaben im Oktober 2019 nur $50 \%$ der Befragten an, dass sie alle erhobenen Befunde der Patienten in die ePA einstellen würden (• Abb. 1).

$\mathrm{Zu}$ fordern ist für die Zukunft, dass für die valide Nutzung der ePA alle relevanten Informationen, z.B. in einem unveränderlichem „Arztbereich“, zur Verfügung stehen. Alternativ wäre an eine Kennzeichnung von Befunden zu denken, die vom Versicherten gesperrt/ gelöscht wurden.

Um Schnittstellenprobleme $\mathrm{zu}$ vermeiden, ist eine gleiche Semantik erforderlich. Eine $100 \%$ ige Kompatibilität zu allen gängigen Praxis-/Klinik-SoftwareSystemen mit einfacher Bedienung sowie maximaler Daten- und Rechtssicherheit, besondere für den Datentransfer zwischen den Akteuren, muss gewährleistet werden. Der Aufwand für die Einstellung und Validierung der Befunde kostet Zeit und bedarf einer adäquaten Vergütung, die durch die geplante einmalige $10-€$ Zahlung derzeit nicht gegeben ist.

\section{Datenschutz}

Mit dem E-Health-Gesetz [35] und dem DVG [36] wurde vom Gesetzgeber ein Fahrplan für den weiteren Ausbau der TI, die Verbesserung der Kommunikation verschiedener IT-Systeme im Gesundheitswesen sowie für die Einführung telemedizinischer Leistungen (z. B. OVS) geschaffen. Hinzu kommen Regelungen für den Zugang zu Gesundheits-Apps so- 


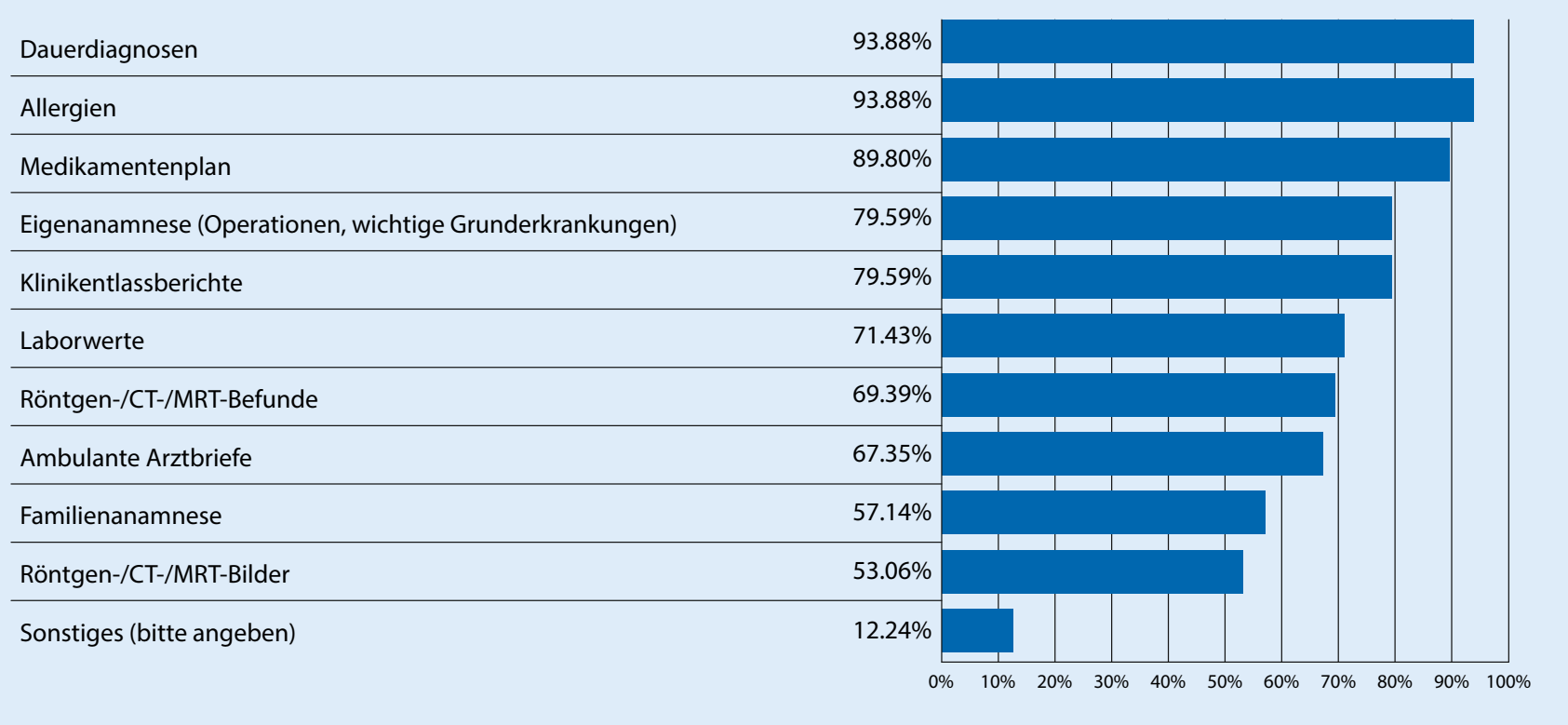

Abb. 1 A Ergebnis der Onlineumfrage des Berufsverbands für Orthopädie und Unfallchirurgie zu den Inhalten eines Maximalstandards in der elektronischen Patientenakte

wie zum Umgang mit digital erhobenen Daten für Forschungszwecke.

Essenzieller Grundpfeiler jeglicher Digitalisierung im Gesundheitswesen ist die Einhaltung der Anforderungen der Datenschutz-Grundverordnung (DSGVO) zum Schutz personenbezogener Daten sowie zur informationellen Selbstbestimmung. Die DSGVO definiert Datenverarbeitungsprozesse, unterstreicht die besondere Stellung von personenbezogenen Daten zu Gesundheit und Genetik und fordert den aktiven Nachweis von Datenschutzmaßnahmen von Unternehmen und Organisationen ein. Nachdruck wird dem Thema mit einem deutlich wehrhaften Bußgeldkatalog verliehen. Jede Praxis, jede Klinik ist verantwortlich und haftbar für Verstöße gegen den Datenschutz in den eigenen vier Wänden und auch dann, wenn Daten (oder Röntgenbilder, Laborproben, Fotos ...) den eigenen Wirkungskreis verlassen. Dabei muss der Tatsache Rechnung getragen werden, dass sich der Begriff , anonymisiert“ erhobener Daten in Zeiten von künstlicher Intelligenz und der Möglichkeit eines viel einfacheren Zugriffs auf riesige Vergleichsdatensätze gewandelt hat. Ein Röntgenbild mit geschwärztem Namen gilt nicht mehr als anonymisiert und darf folglich auch nicht unverschlüsselt oder über unbekannte, beispielsweise ausländische Server versendet werden.

\section{\) Die Beachtung des \\ Datenschutzes schafft Vertrauen in die Digitalisierung des Gesundheitssystems}

Die Beachtung des Datenschutzes sollte trotz des initial erhöhten Mehraufwands nicht als Hindernis empfunden werden, sondern als Chance, in der Bevölkerung Vertrauen für die Digitalisierung des Gesundheitssystems zu schaffen. Nicht umsonst waren $83 \%$ der Teilnehmer einer Versichertenbefragung der Barmer-Krankenkasse [37] zur eGK besorgt um den Schutz ihrer Privatsphäre und den Zugriff von Dritten auf persönliche gesundheitsbezogene Daten.

\section{Resümee und Ausblick}

Ärzte und Patienten stehen in einschlägigen Umfragen Digitalisierungsprojekten offen gegenüber. Die eingesetzten Lösungen müssen praktikabel sein und den Anwendern einen klaren Mehrwert bieten. Dieser Anforderung wird der aktuelle Ausbaustand der TI nicht gerecht.
Sinnvolle Anwendungen sind bislang nur angekündigt.

Viele niedergelassene Kolleginnen und Kollegen bedienen sich frei verfügbarer Alternativangebote wie OVS, Onlineterminvergabe und ePA. Sie stehen der staatlich verordneten Digitalisierung skeptisch gegenüber. In der Klinik ist die digitale Prozessoptimierung bereits weit fortgeschritten. Eine sektorübergreifende digitale Kommunikation mit niedergelassenen Ärzten und von Klinik zu Klinik ist aber noch immer kaum möglich. In $\mathrm{O}$ und $\mathrm{U}$ wird der Einstieg in die Digitalisierung mit einer Reihe von Rahmenverträgen sowie durch Integration in das Patienteninformationsportal Orthinform erleichtert. Mit wenigen Klicks können Onlineterminvergabe, OVS, Ressourcenmanagement usw. gebucht sowie in das persönliche Arztprofil und die eigene Webseite integriert werden [10]. Weiterhin wird der Einstieg in die Digitalisierung durch das Aushandeln von Selektivverträgen unterstützt, die eine bessere Honorierung digitaler Angebote wie beispielsweise der OVS oder der Onlineterminvergabe garantieren. 


\section{Fazit für die Praxis}

Orthopäden und Unfallchirurgen sollten sich weiter aktiv dem Thema widmen und steuernd eingreifen, um die Chancen und Rahmenbedingungen der Digitalisierung für ihre Patienten und im Interesse der Heilkunst zum Einsatz zu bringen.

\section{Korrespondenzadresse}

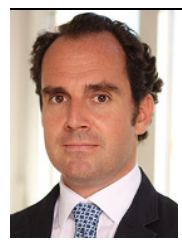

PD Dr. Dominik Pförringer

Klinik und Poliklinik für Unfallchirurgie, Klinikum rechts der Isar, TU München Ismaninger Str. 22, 81675 München, Deutschland dominik.pfoerringer@mri.tum.de

\section{Einhaltung ethischer Richtlinien}

Interessenkonflikt. D. Pförringer, J. Ansorg, G. Osterhoff, F. Dittrich, J. Scherer, U. de Jager und D.A. Back geben an, dass kein Interessenkonflikt besteht.

Für diesen Beitrag wurden von den Autoren keine Studien an Menschen oder Tieren durchgeführt. Für die aufgeführten Studien gelten die jeweils dort angegebenen ethischen Richtlinien.

\section{Literatur}

1. (2019) Empfehlung zum Anschluss an die Telematikinfrastruktur (TI). Bvou Infobr 3:48-49

2. Betriebsarten des Konnektors. Informationsblatt der gematik. https://fachportal. gematik.de/fileadmin/user_upload/fachportal/ files/Service/Anschluss_medizinischer_ Einrichtungen_an_die_Tele-atikinfrastruktur_ DVO_Informationsblatt_BetriebsartenKonnektor_V1.0.0.pdf.Zugegriffen: Mai 2020

3. Eikenberg R (2019) Warum eine komplette Arztpraxis offen im Netz stand. c't 25 Heise Verl. https://www.heise.de/ct/artikel/ Warum-eine-komplette-Arztpraxis-offen-imNetz-stand-4590103.html

4. Sicherheitslücken bei E-Arztausweis und SMC-B aufgedeckt. Ärztezeitung vom 27.12.2019. https://www.aerztezeitung.de/Wirtschaft/ Sicherheitsluecken-bei-E-Arztausweis-undSMC-B-aufgedeckt-405306.html. Zugegriffen: 28. Dez. 2019

5. Schmedt, Michael:Telematikinfrastruktur:Zugang mit Identitätsdiebstahl. Dtsch Arztebl 2020; 117(12):A-7

6. KBV KBV: Versichertenstammdatenmanagement (VSDM). https://www.kbv.de/html/vsdm.php. Zugegriffen:Mai 2020

7. (2019) Gemeinsam für ein digitales. Praxismanagement Bvou Jahresbericht, 50-51. https://www. bvou.net/wp-content/uploads/2016/02/BVOU_ Jahresbericht_2019.pdf
8. Ärztetag beschließt Liberalisierung der Fernbehandlung. Aerzteblatt.de vom 10.05.2018. https://www.aerzteblatt.de/nachrichten/95084/ Aerztetag-beschliesst-Liberalisierung-derFernbehandlung. Zugegriffen: Mai 2020

9. Lembeck, Burkhard: "Check My Back" Selektivvertrag Rückenschmerz gestartet. Orthopädisch-Unfallchirurgische Mitteilungen und Nachrichten 2020; 10 (3), 26-27. Springer Verlag Heidelberg. https://www. springermedizin.de/rueckenschmerz/check-myback-selektivvertrag-rueckenschmerz-gestartet/ 18054146

10. Ansorg J (2018) Reputation und Digitalisierung im Internet mit Orthinform. BVOU. Infobrief, Bd. 4, S8-11

11. Albrecht U-V App-Synopsis - User- Deutsche Version.In:

12. Albrecht U-V (2019) Einheitlicher Kriterienkatalog zur Selbstdeklaration der Qualität von Gesundheits. Apps Ehealth Suisse. https://www.e-healthsuisse.ch/fileadmin/user_upload/Dokumente/D/ kriterienkatalog-selbstdeklaration-gesundheitsapps.pdf

13. Bundesgesetzblatt (2019) Gesetz für eine bessere Versorgung durch Digitalisierung und Innovation (Digitale-Versorgung-Gesetz) Vom 09. Dezember 2019. In:Bundesanzeiger Verlag $\mathrm{GmbH}$, Bundesgesetzblatt Jahrgang 2019 Teil $1 \mathrm{Nr} .49$

14. Dittrich F BS, Harren Ak, Reinecke F, Serong S, Jung J, Back Da, Wolf M, Landgraeber S (2019) Analysis of secure apps for daily clinical use as a German orthopaedic surgeon-searching for the "needle in a haystack". JMIR Preprints. https://doi.org/10. 2196/17085

15. Dittrich F, Busch A, Harren K et al (2019) Apps in clinical use in orthopedics and trauma surgery: The status quo in Germany. Unfallchirurg 122:690-696

16. Duncan SFM, Hendawi TK, Sperling J et al (2015) iPhone and iPad Use in Orthopedic Surgery. Ochsner J 15:52-57

17. Knitza J, Tascilar K, Messner EM et al (2019) German Mobile Apps in Rheumatology: Review and Analysis Using the Mobile Application Rating Scale (MARS). JMIRMhealth Uhealth 7:e14991

18. Stoyanov SR, Hides L, Kavanagh DJ et al (2015) Mobile app rating scale: a new tool for assessing the quality of health mobile apps. JMIR Mhealth Uhealth $3: \mathrm{e} 27$

19. Stroud C (2018) Cambridge Analytica: The Turning Point In The Crisis About. Big Data. https:// www.forbes.com/sites/courtstroud/2018/04/30/ cambridge-analytica-the-turning-point-in-thecrisis-about-big-data/\#5a8eb6a48ecc

20. Statistisches Bundesamt (2019) Statistisches Bundesamt (Destatis). Wirtschaftsrechnungen 2020.https://www.destatis.de/DE/Home/_inhalt. html

21. Wongworawat, M.D., G. Capistrant, and J.M. Stephenson, The Opportunity Awaits to Lead Orthopaedic Telehealth Innovation: AOA Critical Issues. J Bone Joint Surg Am, 2017.99(17): p. e93.

22. Bertelsmann-Stiftung. Video-Sprechstunden. 20152015 [cited 2020; Available from: https://www.bertelsmann-stiftung.de/fileadmin/ files/BSt/Publikationen/GrauePublikationen/ SpotGes_VideoSprechstunde_dt_final_web.pdf. Zugegriffen: Mai 2020

23. Sathiyakumar, V., et al., Prospective randomized controlled trial using telemedicine for follow-ups in an orthopedic trauma population: a pilot study. J Orthop Trauma, 2015.29(3):p. e139-45.

24. Parks MT, Wang Z, Siu KC (2019) Current Low-Cost Video-Based Motion Analysis Options for Clinical
Rehabilitation: A Systematic Review. Phys Ther 99(10):1405-1425

25. Vergütungsübersicht Videosprechstunde im EBM. https://www.kbv.de/media/sp/ Videosprechstunde_uebersicht_Verguetung. pdf.Zugegriffen: Mai 2020

26. PKV: Ärzte erhalten Extravergütungen in der Coronakrise. aerzteblatt.de vom 13.05.2020. https://www.aerzteblatt.de/nachrichten/112806/ PKV-Aerzte-erhalten-Extraverguetungen-in-derCoronakrise. Zugegriffen: 13. Mai 2020

27. Ansorg J (2019) Videosprechstunden in O\&U: Kann man oder muß man? in Orthopädische. Nachrichten, Bd. 04. Biermann Verlag, Köln, S34-35

28. Storm A et al (2019) Digitalisierungsreport der. DAK, Was Ärzte über die Digitalisierung des Gesundheitssystems denken. medhochzwei Verlag $\mathrm{GmbH}$, Heidelberg

29. Bundesgestzblatt Jahrgang 2019 Teil I Nr. 18, 10. Mai 2019, 646-691. www. bundesgesundheitsministerium.de/fileadmin/ Dateien/3_Downloads/Gesetze_und_ Verordnungen/GuV/T/TSVG_BGBL.pdf

30. www.kbv.de/html/epa.php, Stand:23. Apr. 2019

31. www.bundesgesundheitsministerium.de/pdsg html (Pressemitteilung: „Kabinett beschließt Patientendaten-Schutz-Gesetz", Stand 1. Apr. 2020)

32. www.bundesgesundheitsministerium.de/ fileadmin/Dateien/3_Downloads/Gesetze und_Verordnungen/GuV/P/Gesetzentwurf Patientendaten-Schutz-Gesetz_-_PDSG.pdf (Stand 31. März 2020)

33. Interview mit Ferdinand Gerlach am 28. Febr. 2020 Ärzte Zeitung: www.aerztezeitung.de/Politik/ Datenschutz-darf-keine-Leben-kosten-407161. html

34. de Jager U (2019) Online-Umfrage zur ePA. BVOU, Bd. 10

35. (2020) E-Health-Gesetz. https://www. bundesgesundheitsministerium.de/service/ begriffe-von-a-z/e/e-health-gesetz.html. Zugegriffen: Mai 2020

36. (2019) Digitale-Versorgung-Gesetz. https://www. bundesgesundheitsministerium.de/digitaleversorgung-gesetz.html.Zugegriffen:Mai 2020

37. (2010) Nutzen und Akzeptanz von elektronischen Gesundheitsakten. Abschlussbericht zum Forschungsvorhaben der BARMER GEK. https://www.barmer.de/blob/33814/ a5673f3d2d75bf73e799de68b17cc5c5/data/ abschlussbericht.pdf.Zugegriffen: Mai 2020 